\title{
Design of a Single and Dual Hybrid Piezoelectric Motors
}

\author{
Yung Ting, Kai-Chao Chang, Chih-Hsuan Yu and Amelia Sugondo \\ Department of Mechanical Engineering, Chung Yuan Christian University, No. 200, Chung Pei Rd., Chung Li \\ 32023, Taiwan.
}

\begin{abstract}
(Received 18 July 2019; accepted 19 December 2019)
In this paper, a new hybrid piezoelectric motor was developed. A range of degeneracy was found to obtain multiple choice of resonance frequency by adjusting the length of the elastic blocks neighboring to the longitudinal and torsional vibrators. A template was implemented in between the longitudinal and torsional vibrators to decrease the mutual vibration effect that effectively makes the calculated and actual driving frequency nearly close. Such configuration was instrumental to preserve the expected performance and reduce the trial-and-error design overhaul process of the elastic blocks. Moreover, a dual hybrid motor was easily formed by butting each stator of the two hybrid motors together with a rotor in the middle. Case studies of both single and dual hybrid motors are presented with both simulation and experimental results to demonstrate the structure design and the degeneracy approach. Besides the convenience of structurally combining two single hybrid motors to enhance the output, it was interesting to find out whether the proposed dual hybrid motor can significantly increase either the speed or torque by using different phase control methods. Therefore, using the dual hybrid motor provides a great advantage by providing an option for pursuing higher speed or larger torque output for certain applications.
\end{abstract}

\section{INTRODUCTION}

Langenvin type transducer using piezo ceramics to generate longitudinal vibration was developed several decades ago and successfully used for numerous applications. Ueha et al. were probably the first ones to develop a hybrid transducer like a rotary motor generating rotation without auxiliary transmission mechanisms. ${ }^{1}$ In general, the stator of the transducer is comprised of two types of piezoelectric elements to excite the torsional and longitudinal vibration and generate rotation via appropriate control command. Characteristics and design method of the hybrid transducer was introduced later. ${ }^{2,3}$ Degeneracy that makes the same driving resonant frequency for both the longitudinal and torsional vibrators is an important issue in design. Several methods were developed for degeneracy. To name a few, Lin et al. designed an exponential horn. By choosing an appropriate cross section of the curve shape of the horn, degeneracy was achieved. ${ }^{4}$ Tomikawa et al. reduced the cross-section of the rod so that the resonance frequency of the longitudinal vibration is largely reduced to match the unchanged low resonance frequency of the torsional vibration. ${ }^{5}$ They also proposed to use a step-formed vibrator to increase the resonance frequency of the longitudinal vibration but decrease the torsional vibration so that degeneracy could be achieved. Wang and Guo developed an inner adjusting ring. By changing the internal radius or position of the inner adjusting ring, resonance frequency of the radial and torsional vibrators was matched. ${ }^{6}$ Yi et al. used an adjusting ring encircled on the transducer to change resonant frequency of the torsional vibrator so that it would match the longitudinal one. Two sets of longitudinal and torsional vibrators that are symmetrically implemented on the sides of the middle rotor was developed to construct an ultrasonic motor for improvement of the output power. ${ }^{7}$ A few of approaches attempting to improve the performance of the hybrid transducer-type motor were proposed. For instance, Satonobu et al. proposed a hybrid ultrasonic motor comprised of two groups of longitudinal and torsional vibrators equally separated by a flange and clamped by a central bolt. With increase of longitudinal vibration, larger torque is gained. Suitable driving frequency for both the longitudinal and torsional vibrators was chosen via a range of degeneracy. ${ }^{8}$ Ishii et al. used thrust bearing with lubricant to increase the output performance. ${ }^{9}$ Qiu et al. improved the efficiency significantly of the hybrid transducer-type motor by using lubricant, in particular at high preload. Lifetime of the hybrid motor is extended due to well protection of the contact surface. ${ }^{10}$ Zippo et al. investigated the nonlinear dynamics of circular cylindrical shells by using numerical simulations. On the condition of high environmental temperature leading to the shell, it was found that the natural frequency would decrease while temperature increased. ${ }^{11,12}$ In this study, a stator was constructed with a template employed in between the longitudinal and torsional vibrators as well as their associated elastic blocks. Via the adjustment of the length of the elastic blocks neighboring to the longitudinal and torsional vibrators, a range of degeneracy is obtained to provide multiple choices of resonance frequency. The template works effectively to avoid significant difference between the calculated resonance frequency and the actual driving frequency. Also, to butt two stators together with a middle rotor employed in between them is proposed for larger output. It was found that not only can this preserve a range of degeneracy but also it can increase either torque or speed output performance by using different control commands. By using the same phase control command for both stators, they will gain higher torque; on the contrary, using the reverse phase control command, they will gain larger speed. This was such an interesting characteristic of the developed dual hybrid motor; it allows the user to choose either higher torque or speed output fit for the application demand.

\section{STRUCTURE OF SINGLE/DUAL HYBRID MOTORS}

In Fig. 1 1(a), the stator of the proposed single hybrid motor is constructed with cylindrical longitudinal (PZT-L) and torsional (PZT-T) ceramic vibrators. Using pivot and thread as 


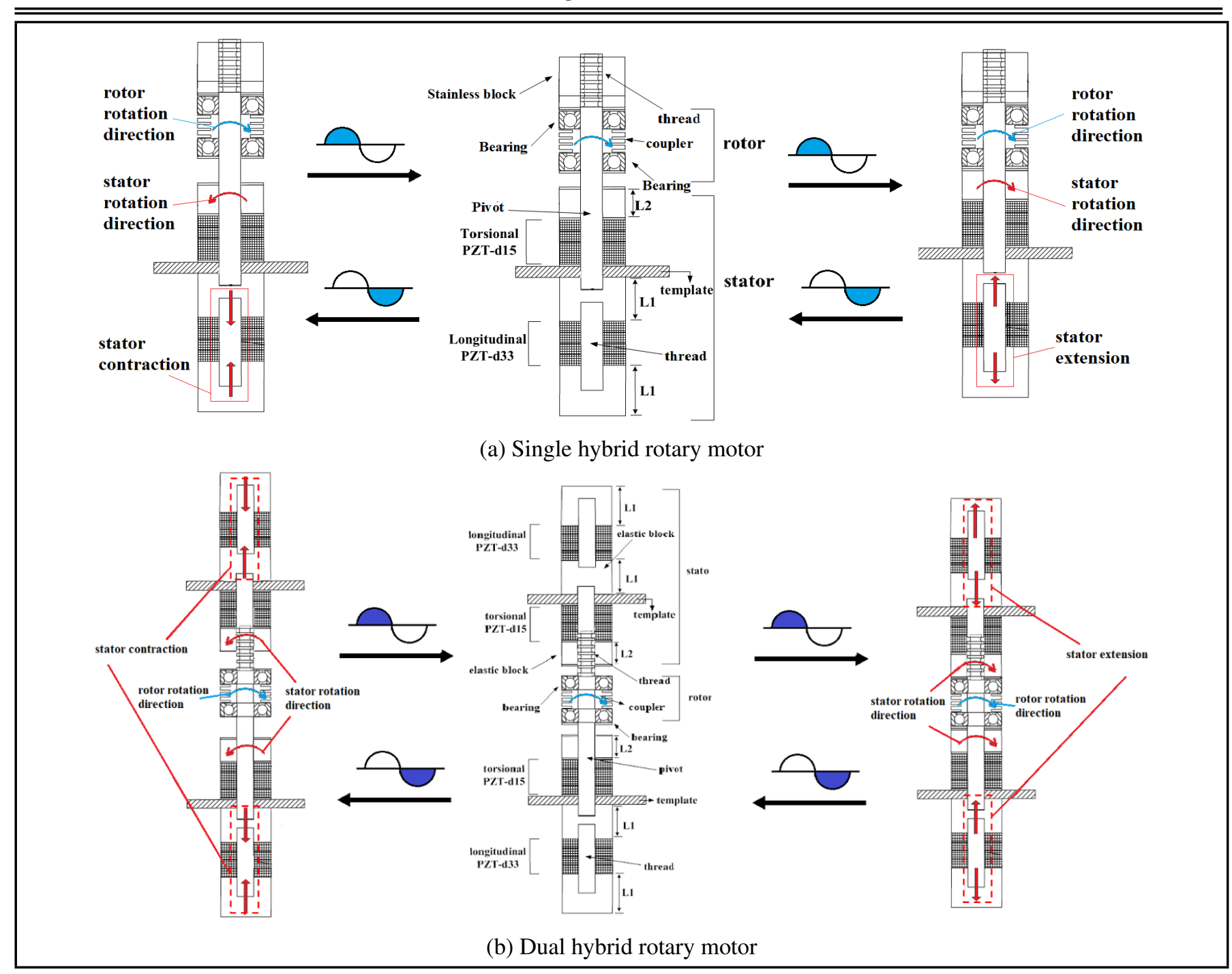

Figure 1. Structure of the hybrid single/dual rotary motor

well as related mechanisms, the entire structure is fixed with a preload force. Different from the usually seen hybrid rotary motor, a template is assigned between the longitudinal and torsional ceramic actuators. This type of dynamic-decoupled structure is instrumental to easily adjust for a common resonance frequency in degeneracy process due to reduction of the mutual vibration propagation. The longitudinal ceramic vibrators are basically made of $d 33$ associated with two equal elastic blocks L1. The torsional ceramics vibrators are made of $d 15$, which is polarized in the circumferential direction and applied electric voltage in the longitudinal (axial) direction. These two types of ceramics vibrators are excited independently but with the same driving frequency. As the longitudinal vibrator extends, contact of the rotor occurs. Meanwhile, the torsional ceramics generates rotary displacement, so the rotor is driven to rotate. As the longitudinal vibrator shortens, contact of the rotor is removed. In the meantime, the torsional vibrator is reversed back, and there is no reverse rotation in this period. With appropriate control methods, the above operating method can be achieved so continuous rotation is obtained. In ideal conditions, maximum output is gained with $90^{\circ}$ phase difference between the longitudinal and torsional vibrators. ${ }^{2}$ A dual rotary motor is illustrated in Fig. 1(b). Though it is similar to a single hybrid motor, the two stators are butted together with a rotor in the middle. In this study, a shaft coupler, for example, was used as the rotor.

\section{DEGENERACY APPROACH}

Degeneracy is the fundamental task in search of a common resonance frequency for both the longitudinal and torsional vibrators. However, achieving the purpose of degeneracy is not easily accomplished, and there usually is only one solution for the traditional hybrid motor. In the proposed structure, degeneracy was easier to achieve by adjusting the dimension of the longitudinal and torsional blocks. The finite element analysis was used for calculation of the vibration mode. The longitudinal and torsional resonance frequency was independently searched without considering the possible mutual effect. This may be a determinant factor to cause a large difference between the calculated and the actual driving frequency, which in either case, results in undesirable performance or causes tedious trialand-error redesigning process of the elastic block. Thus, using a template to isolate the vibration of the longitudinal vibrators and the torsional vibrators was investigated to see if this would prevent a large deviation of the actual driving frequency from the calculated resonance frequency. As depicted in Fig. 11 , an example of the length of elastic block of L1 and L2 attached to the longitudinal vibrator and torsional vibrators respectively was investigated for a single hybrid motor. Concerning design of the elastic blocks L1 and L2 in practice, length of L1 and L2 are assigned in the range of $15 \sim 28 \mathrm{~mm}$ and $4 \sim 24 \mathrm{~mm}$ respectively. Modal analysis was carried out by using ANSYS for the longitudinal and torsional vibrators respectively. Ce- 
Table 1. Material properties of ceramic vibrator and elastic block

\begin{tabular}{||c|c|c|}
\hline Material properties & Ceramics PZT-8 & Elastic blocks \\
\hline Young's modulus $E(\mathrm{Gpa})$ & 91 & 73 \\
\hline Poisson's ratio $\nu$ & 0.31 & 0.33 \\
\hline Density $\rho\left(\mathrm{kg} / \mathrm{m}^{3}\right)$ & 7600 & 2700 \\
\hline Outer diameter $(\mathrm{mm})$ & 25 & 25 \\
\hline Inner diameter $(\mathrm{mm})$ & 10 & 10 \\
\hline
\end{tabular}

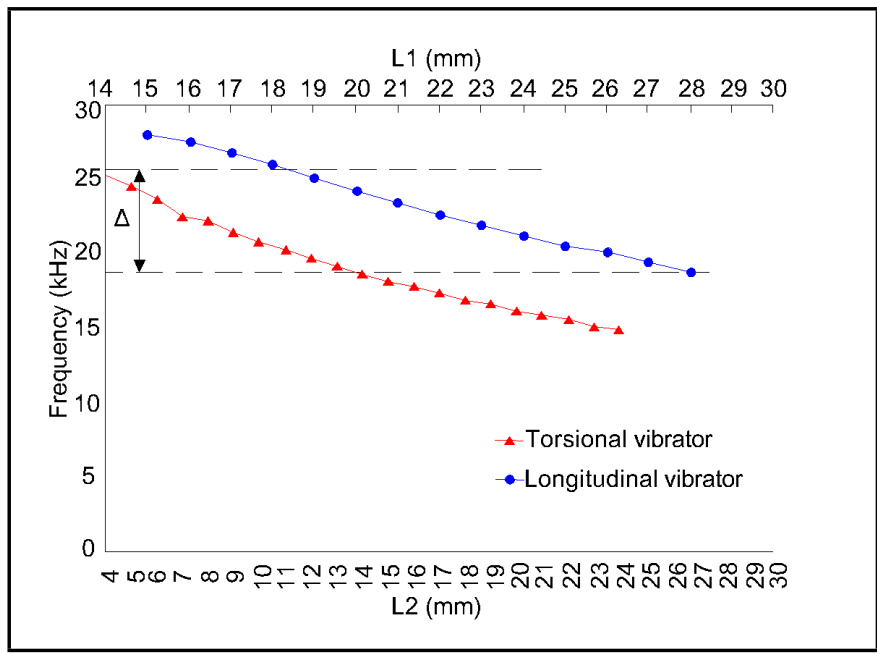

Figure 2. Degeneracy range $\Delta$ for the single hybrid motor

ramics PZT-8 and aluminum alloy were used for the vibrators and elastic blocks respectively. Material properties are listed in Table 1. The piezoelectric matrix, the stiffness matrix and the dielectric matrix used for simulation are given by:

Stiffness matrix

$$
\begin{aligned}
& {\left[S^{E}\right]=} \\
& {\left[\begin{array}{cccccc}
10.1 & -3.03 & -3.03 & 0 & 0 & 0 \\
-3.03 & 10.1 & -3.03 & 0 & 0 & 0 \\
-3.03 & -3.03 & 10.1 & 0 & 0 & 0 \\
0 & 0 & 0 & 26.3 & 0 & 0 \\
0 & 0 & 0 & 0 & 26.3 & 0 \\
0 & 0 & 0 & 0 & 0 & 26.3
\end{array}\right]} \\
&
\end{aligned}
$$

Piezoelectric matrix

$$
[d]=\left[\begin{array}{cccccc}
0 & 0 & 0 & 265 & 330 & 0 \\
0 & 0 & 0 & 330 & 265 & 0 \\
-97 & -97 & 225 & 0 & 0 & 0
\end{array}\right]\left(\times 10^{-12} \mathrm{C} / \mathrm{N}\right)
$$

Dielectric matrix

$$
\left[\epsilon^{T}\right]=\left[\begin{array}{ccc}
1000 & 0 & 0 \\
0 & 1000 & 0 \\
0 & 0 & 1290
\end{array}\right]\left(\times 10^{-12} \mathrm{C} / \mathrm{N}\right)
$$

Note that, the $1^{\text {st }}$ mode is usually used in design; however, the $2^{\text {nd }}$ mode or even a higher mode with a higher frequency is likely used to gain higher output performance. In this study, the $1^{\text {st }}$ modes, for instance, were ascertained to search for resonance frequency of both the longitudinal and resonant vibrators with respect to different elastic block length L1 and L2, which are listed in Table 2.

In Fig. 2, degeneracy range $\Delta$ is found in the frequency of $18 \sim 25 \mathrm{kHz}$ corresponding to the L1 and L2 with length in the range of $18 \sim 28 \mathrm{~mm}$ and $4 \sim 14 \mathrm{~mm}$ respectively.

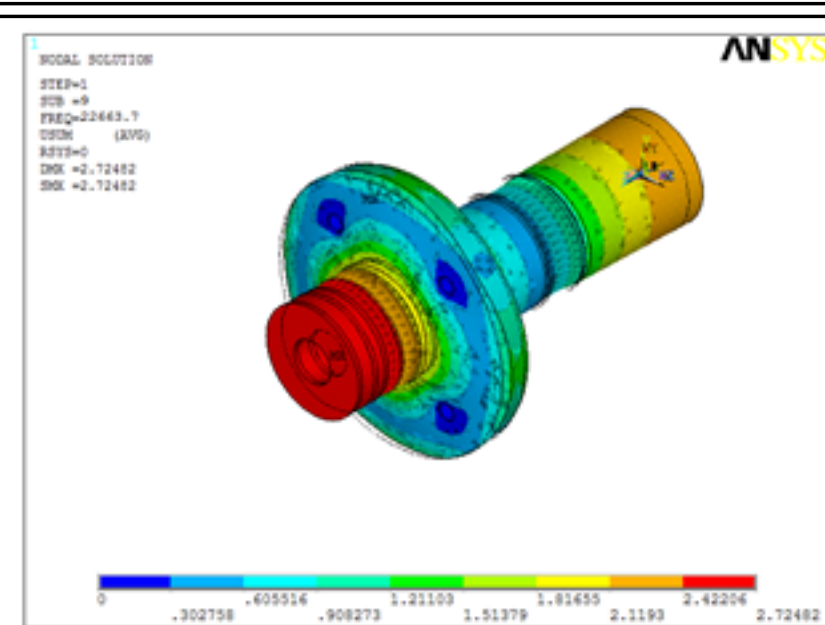

(a) Longitudinal vibrator

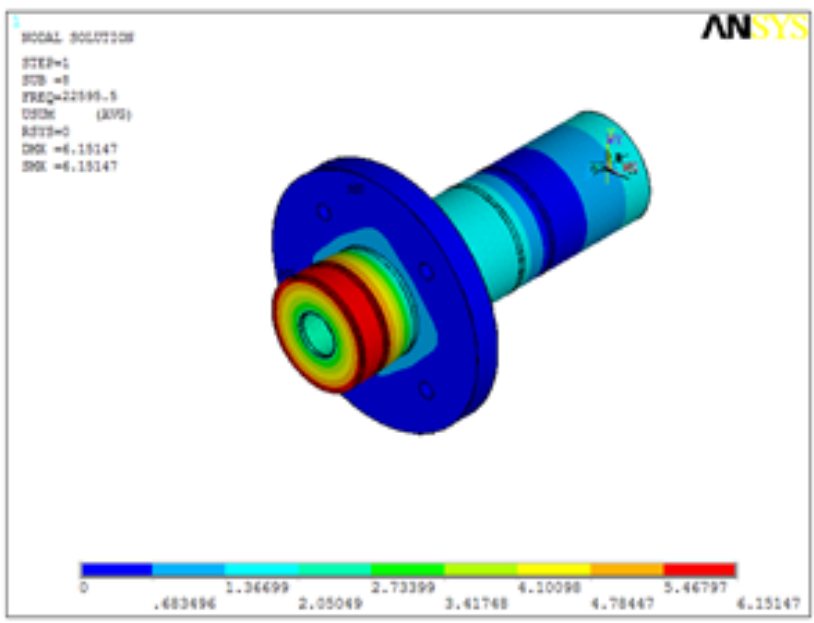

(b) Torsional vibrator

Figure 3. Modal analysis for the single hybrid motor with $(\mathrm{L} 1, \mathrm{~L} 2)=(23,7) \mathrm{mm}$

Table 2. Resonance frequencies of the single hybrid motor

\begin{tabular}{||c|c|c|c|c|c||}
\hline L1 & $\begin{array}{c}\text { Resonance } \\
\text { frequency } \\
(\mathrm{mm})\end{array}$ & $\begin{array}{c}\text { L2 } \\
\text { (longitudinal) }\end{array}$ & $\begin{array}{c}\text { Resonance } \\
\text { frequency } \\
(\mathrm{mm})\end{array}$ & $\begin{array}{c}\text { L2 } \\
\text { (torsional) }\end{array}$ & $\begin{array}{c}\text { Resonance } \\
\text { frequency } \\
(\mathrm{mm})\end{array}$ \\
\hline (torsional) \\
\hline 15 & 28029.7 & 4 & 25504.5 & 18 & 16950.5 \\
\hline 16 & 27554.7 & 5 & 24568.8 & 19 & 16574.6 \\
\hline 17 & 26870.5 & 6 & 23708.9 & 20 & 16240.8 \\
\hline 18 & 26040.6 & 7 & 22595.3 & 21 & 15846.4 \\
\hline 19 & 25279.9 & 8 & 22179.9 & 22 & 15523.3 \\
\hline 20 & 24421.8 & 9 & 21496.1 & 23 & 15210.1 \\
\hline 21 & 23896.6 & 10 & 20858.3 & 24 & 14909.2 \\
\hline 22 & 23296.6 & 11 & 20261.6 & N/A & N/A \\
\hline 23 & 22663.6 & 12 & 19701.8 & N/A & N/A \\
\hline 24 & 21263.6 & 13 & 19175.5 & N/A & N/A \\
\hline 25 & 20606.4 & 14 & 18679.5 & N/A & N/A \\
\hline 26 & 20155.9 & 15 & 18211.2 & N/A & N/A \\
\hline 27 & 19397.3 & 16 & 17768.16 & N/A & N/A \\
\hline 28 & 18840.3 & 17 & 17348.1 & N/A & N/A \\
\hline
\end{tabular}

In Fig. 3, an example of $(L 1, L 2)=(23,7) \mathrm{mm}$ is selected to show the ANSYS modal analysis. It is seen that the resonance frequency of 22.63 and $22.59 \mathrm{kHz}$ for both longitudinal and torsional vibrators are nearly the same. Similarly, modal analysis was investigated for the dual hybrid motor constructed with two single hybrid motors. Resonance frequencies of the longitudinal and torsional vibrator are found and listed in Table 3 with respect to different elastic block length L1 and L2 in the range of $17 \sim 25 \mathrm{~mm}$ and $4 \sim 13 \mathrm{~mm}$ respectively. 
Table 3. Resonance frequencies of the dual hybrid motor

\begin{tabular}{||c|c|c|c||}
\hline $\begin{array}{c}\text { L1 } \\
(\mathrm{mm})\end{array}$ & $\begin{array}{c}\text { Resonance frequency } \\
(\mathrm{Hz}) \\
(\text { longitudinal })\end{array}$ & $\begin{array}{c}\text { L2 } \\
(\mathrm{mm})\end{array}$ & $\begin{array}{c}\text { Resonance frequency } \\
(\mathrm{Hz}) \\
\text { (torsional) }\end{array}$ \\
\hline 14 & 25300 & 4 & 22500 \\
\hline 15 & 24300 & 5 & 21500 \\
\hline 16 & 23300 & 6 & 20900 \\
\hline 17 & 22500 & 7 & 20000 \\
\hline 18 & 21900 & 8 & 19264 \\
\hline 19 & 21502 & 9 & 19000 \\
\hline 20 & 20900 & 10 & 18800 \\
\hline 21 & 20260 & 11 & 18600 \\
\hline 22 & 19733 & 12 & 18100 \\
\hline 23 & 19413 & 13 & 17700 \\
\hline 24 & 18462 & 14 & N/A \\
\hline 25 & 17300 & 15 & N/A \\
\hline
\end{tabular}

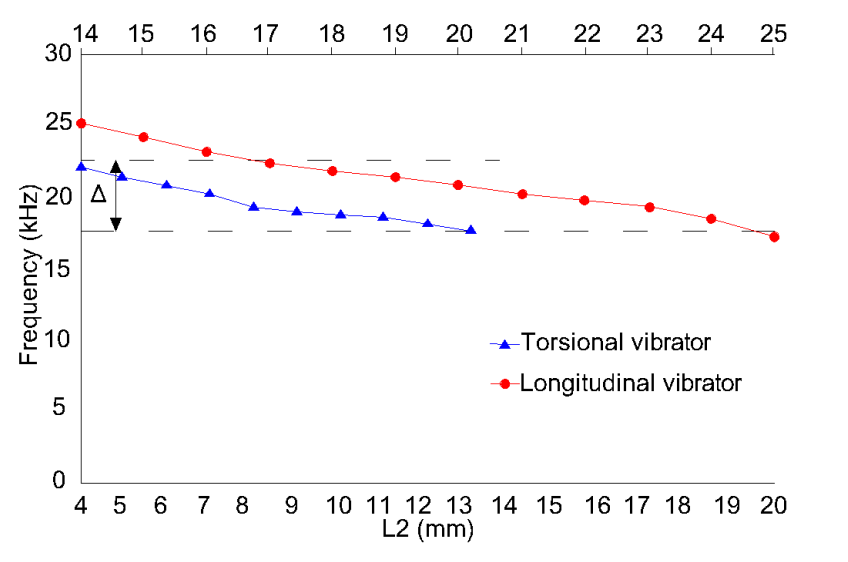

Figure 4. Degeneracy range $\Delta$ for the dual hybrid motor

In Fig. 4, degeneracy range $\Delta$ is found in the frequency of $19 \sim 23 \mathrm{kHz}$ corresponding to the L1 and L2 with length. Butting the two single hybrid motors with elastic block length $(L 1, L 2)=(23,7) \mathrm{mm}$ forming a dual hybrid motor, nearly the same resonance frequency of 19.41 and $20 \mathrm{kHz}$ is searched for in both longitudinal and torsional vibrators respectively for the dual hybrid motor as shown in Fig. 5.

\section{EXPERIMENT}

Via degeneracy process, a range of resonance frequency was found for both single and dual hybrid motors. As described above, the example of elastic block length $(L 1, L 2)=$ $(23,7) \mathrm{mm}$ was chosen for building both single and dual hybrid motors to compare their performance. Figure 6(a) shows the structure of a single hybrid motor. Using an impedance analyzer (Angilent 4294A), the resonance frequency of the single hybrid motor was measured about 22.38 and $22.94 \mathrm{kHz}$ for longitudinal and torsional vibrators respectively and shown in Fig. 7. It was found that the error between the analytical simulation of $22.63,22.59 \mathrm{kHz}$ and experimental measurement of resonance frequency was almost negligible, which indicates both results were in good approximation. To butt the two single hybrid motors with elastic block length $(L 1, L 2)=$ $(23,7) \mathrm{mm}$ for each one to form a dual hybrid motor is shown in Fig. 6(b).

Resonance frequency estimated by ANSYS and measured at the chosen signal curve valley by an impedance analyzer was 20.28 and $20.06 \mathrm{kHz}$ for longitudinal and torsional vibrator respectively and shown with a red circle in Fig. 8. Error between the analytical simulation of $19.41,20 \mathrm{kHz}$ and experimental measurement of resonance frequency was very small. By fol-

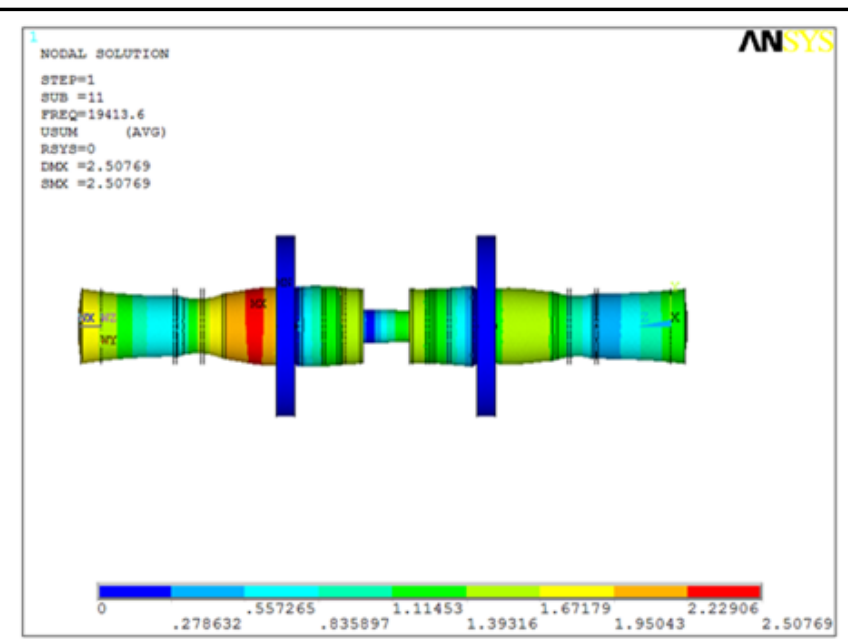

(a) Longitudinal vibrator

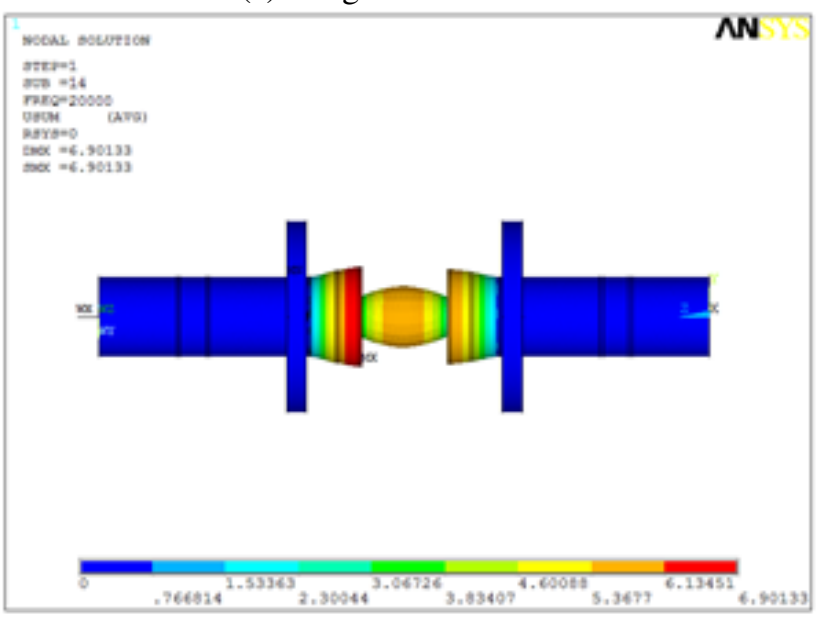

(b) Torsional vibrator

Figure 5. Modal analysis for the dual hybrid motor with $(\mathrm{L} 1, \mathrm{~L} 2)=(23,7) \mathrm{mm}$

lowing the measured frequency with an impedance analyzer, the single and dual hybrid motor are driven in practice with the actual frequency of about 22.8 and $19.2 \mathrm{kHz}$ respectively. The difference between the calculated and actual driving frequency of both the single and dual hybrid motors was minor. In these case studies, degeneracy was successfully reached for both the single and dual hybrid motors. Also, using a template to separate the longitudinal and torsional vibrators was verified to achieve the purpose of approximating the calculated resonance frequency to the actual driving frequency. Evidence has shown that a control command with a $90^{\circ}$ phase difference between the longitudinal and torsional ceramic vibrators will gain maximum output, which is applied to the single and dual hybrid motors respectively. Further investigation of control command was embarked on the dual hybrid motor. Although control command with a $90^{\circ}$ phase difference was driven to each stator comprising of longitudinal and torsional ceramic vibrators, very interesting and attractive outcomes of gaining different torque and speed output were found while using different phases of the control command between the two sets of stators. In Fig. 9(a), both stators are driven with the same phase control command. In Fig. 9(b), both stators are driven with the same control command but with reverse phase. That is, one stator was employed with $V_{L}$ and $V_{T}$, and the other one $V_{L}^{\prime}$ and $V_{T}^{\prime}$. As seen in Fig. 9, the rotor is driven in only half the period but pressed with both longitudinal vibrators while using same phase control method; however, the rotor was driven in 


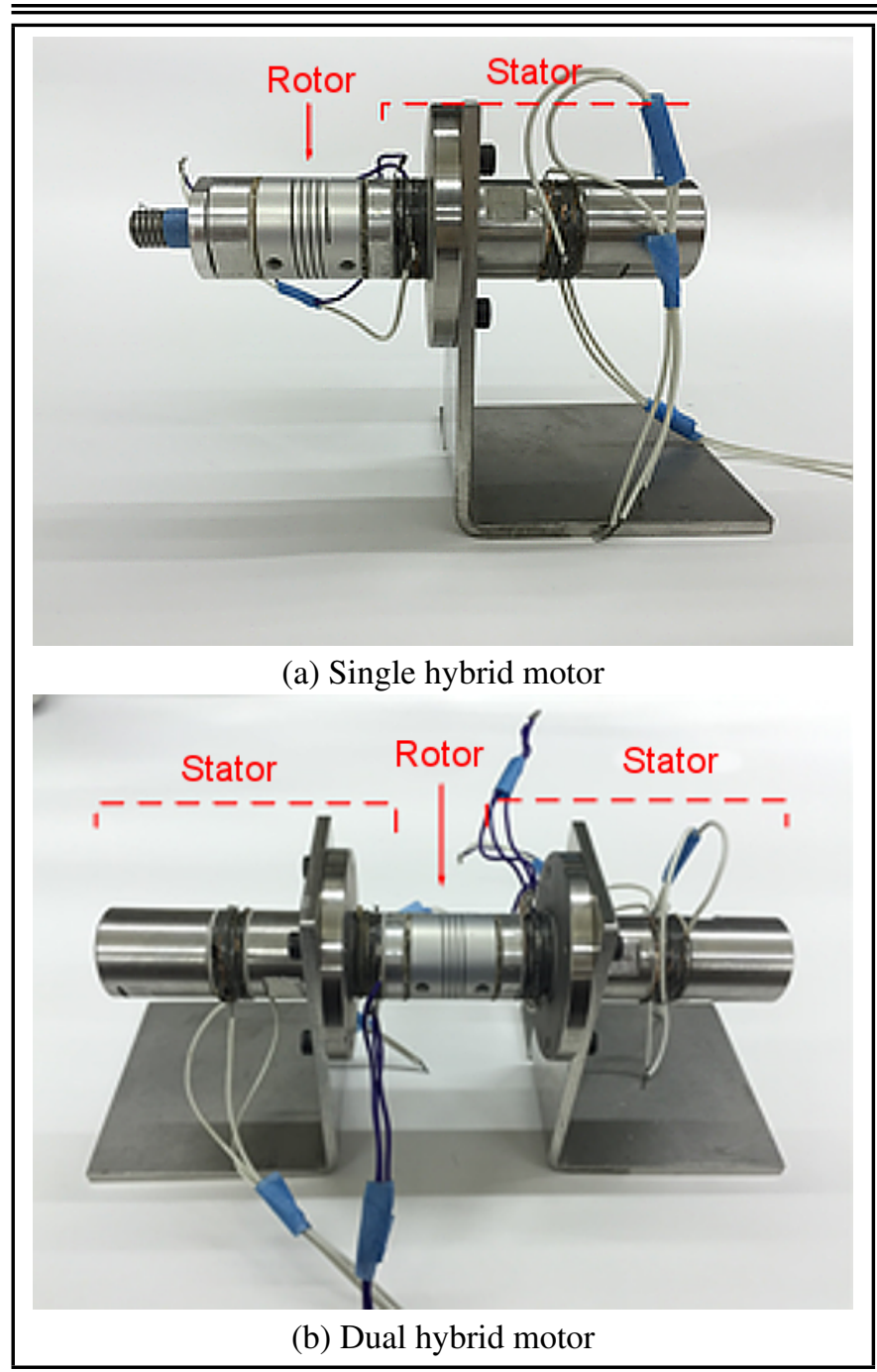

Figure 6. Photographs of the single and dual hybrid rotary motor

the full period but pressed with only one longitudinal vibrator while using the reverse phase control method.

Maximum speed and torque were measured by torque transducer KYOWA TP-20KCE associated with photoelectric sensor PZ-G41B. The experiment with applied voltage of $80,100,120$, and $150 \mathrm{~V}$ respectively was carried out for the three cases: single hybrid motor, the same phase control and the reverse phase control commands of the butted dual hybrid motors. The average value of six experimental results of the maximum torque and speed are highlighted in Figs. 10-12 respectively. Using torque transducer HBM T22, further testing of dynamic torque vs. speed with an example of applied voltage $150 \mathrm{~V}$ for three cases are shown in Fig. 13.

Maximum speed and torque reached 25,35, $46 \mathrm{rpm}$ and $0.208,0.354,0.251 \mathrm{Nm}$ for (single, same phase control/dual, reverse phase control/dual) hybrid motors respectively. Compared to the single hybrid motor, as expected, nearly double the maximum torque and speed output was gained for the same phase control method and reverse phase control method respectively. Besides, much greater torque or speed than that of the single hybrid motor appears almost in every operating condition. Moreover, though the stator presses and drives the rotor in only half the period, same as the single hybrid motor, the torque was greatly increased as well and the speed was apparently increased while using the same phase control. It may come from both longitudinal vibrators concurrently pressing the rotor to provide more contact force to move the rotor. In

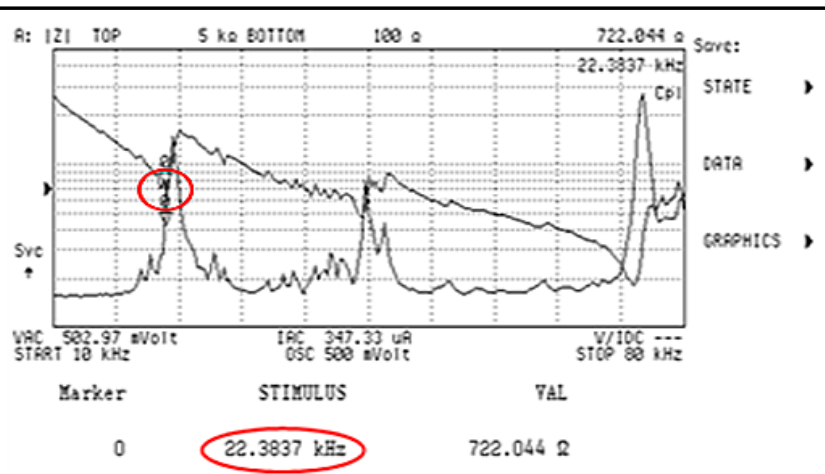

(a) Longitudinal vibrator

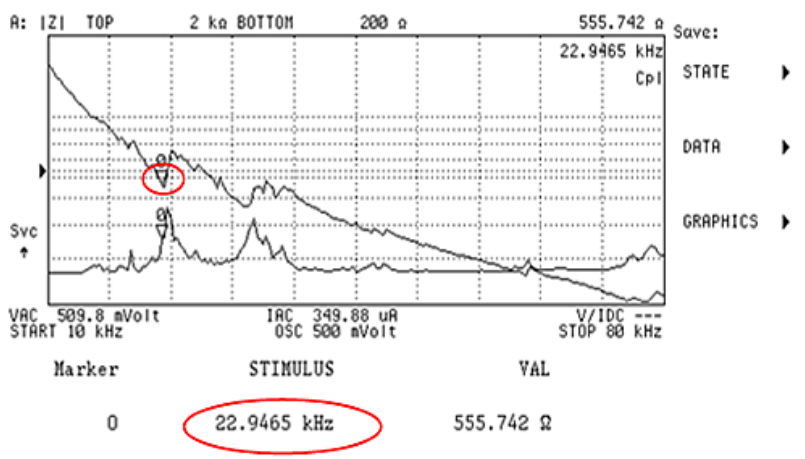

(b) Torsional vibrator

Figure 7. Resonance frequency measured in an impedance analyzer for the single hybrid motor

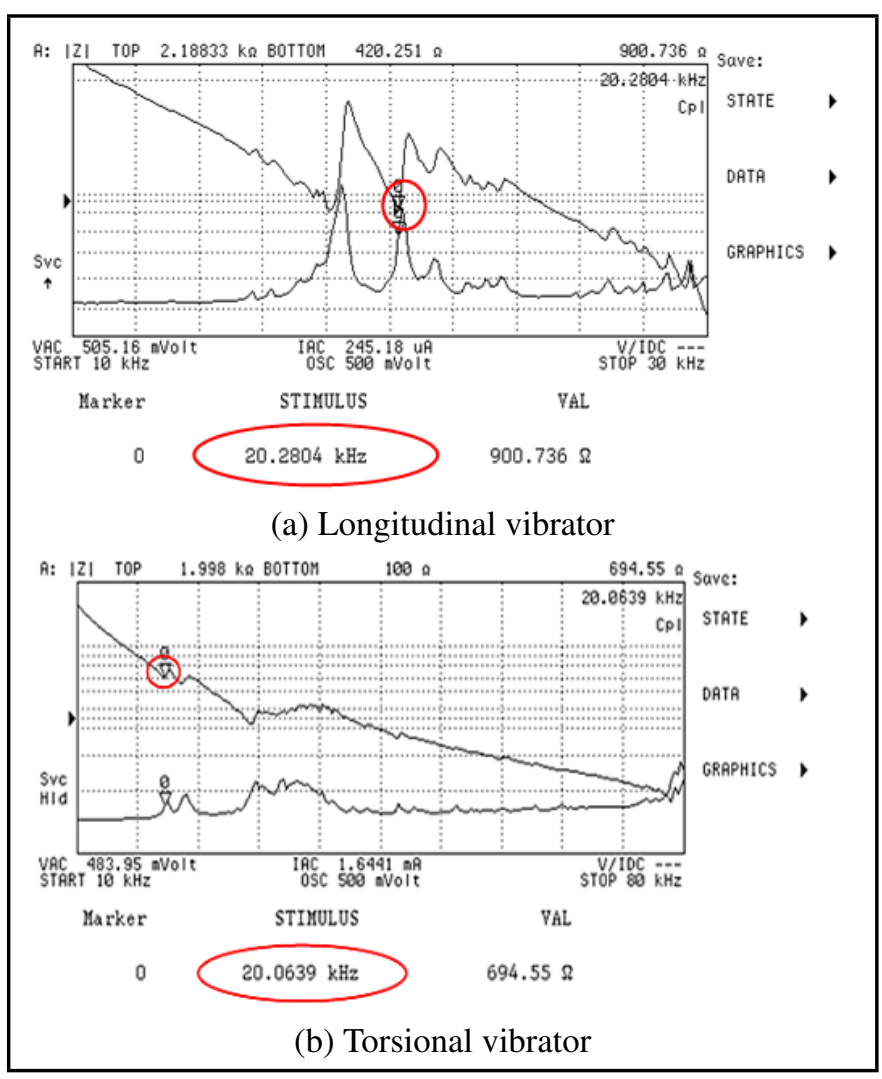

Figure 8. Resonance frequency measured in an impedance analyzer for the dual hybrid motor 


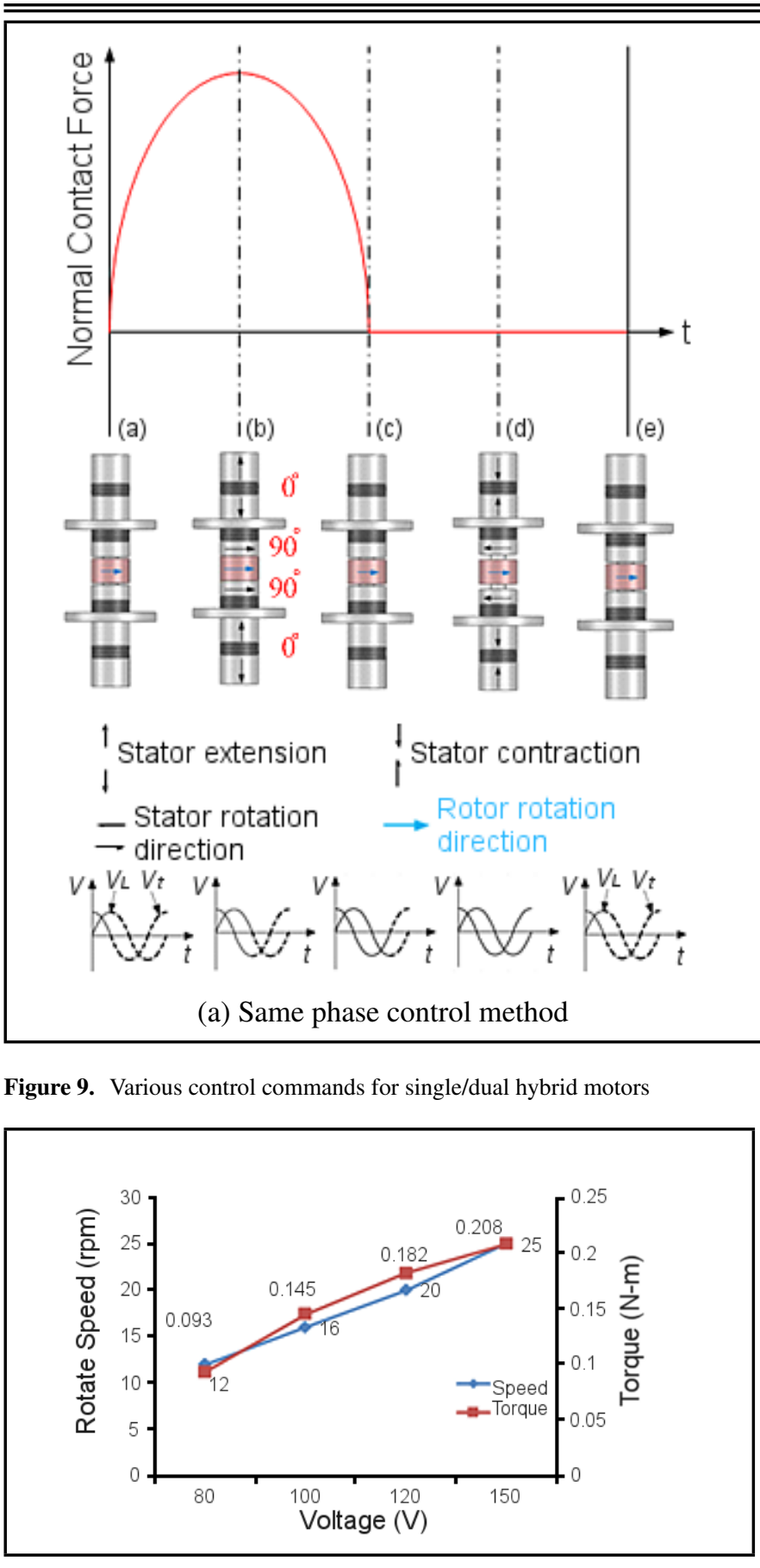

Figure 10. Maximum torque and speed as a function of applied voltage

(single motor)

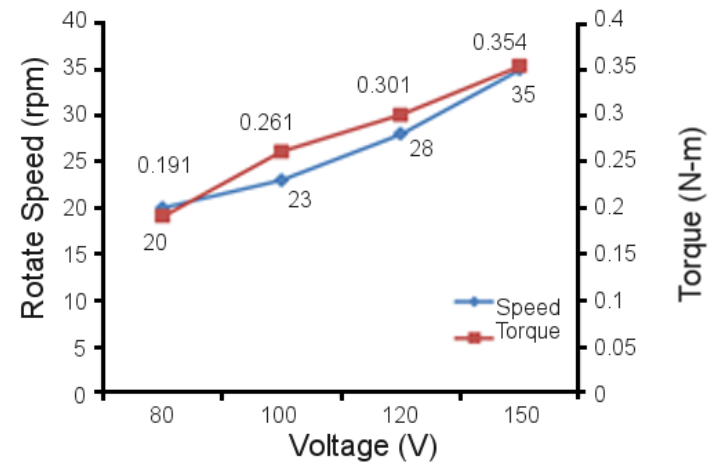

Figure 11. Maximum torque and speed as a function of applied voltage (dual hybrid motor-same phase control)
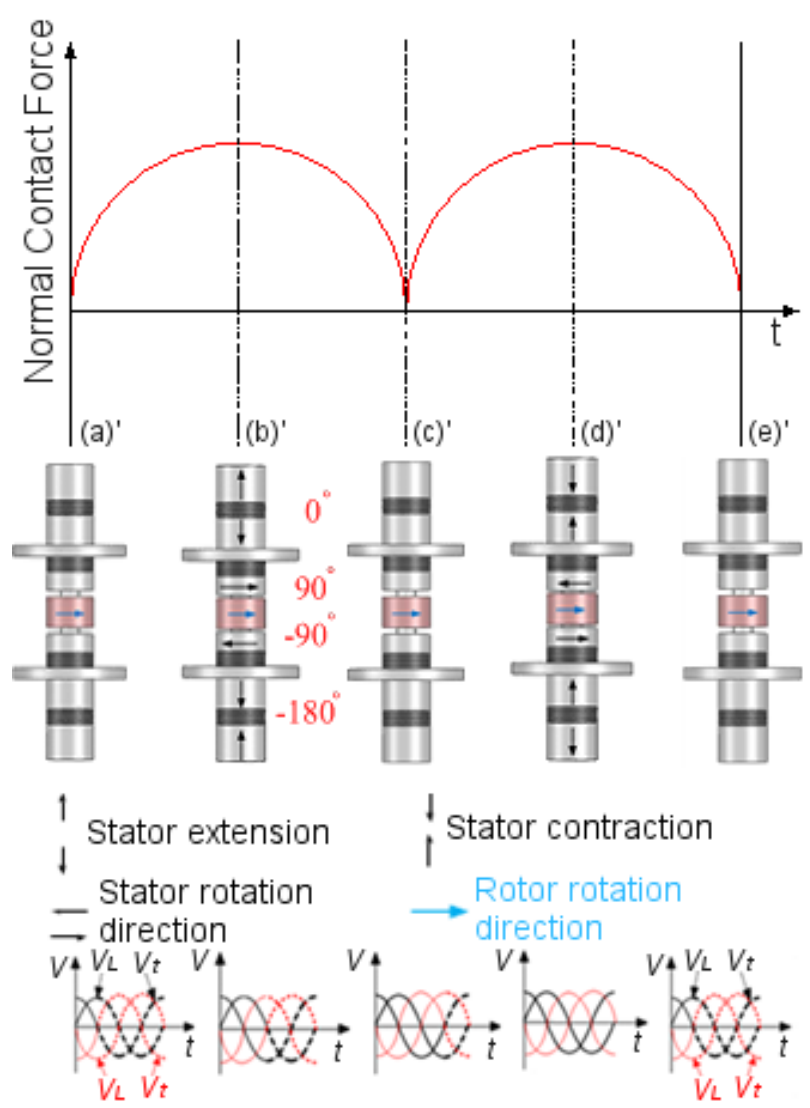

(b) Reverse phase control method

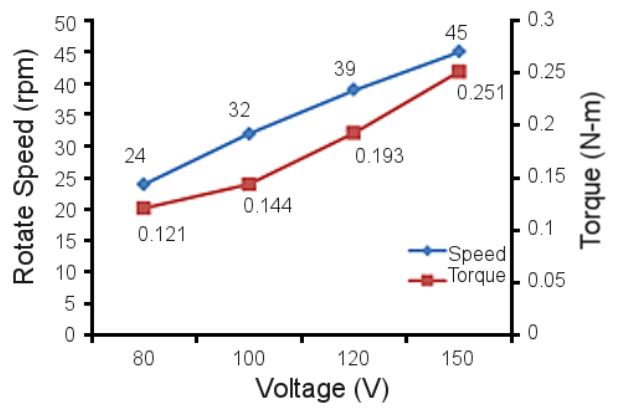

Figure 12. Maximum torque and speed as a function of applied voltage (dual hybrid motor-reverse phase control)

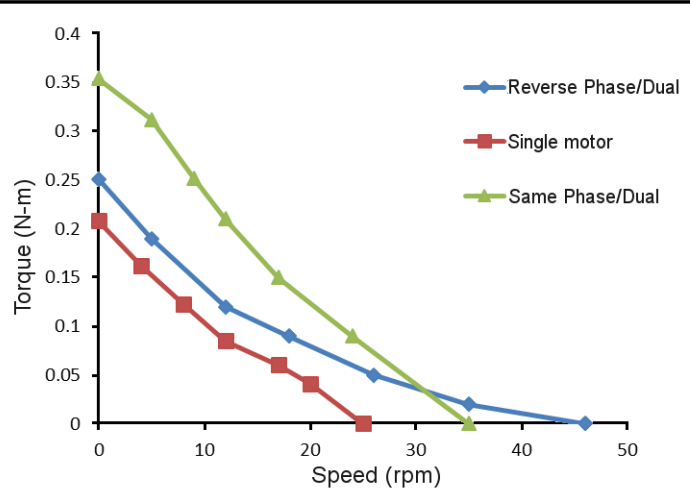

Figure 13. Torque as a function of speed for the single and dual hybrid motors 
other words, large longitudinal deformation was instrumental to generate more output. ${ }^{8}$ To sum up, the dual hybrid motor operating with the same phase control method was able to gain much larger torque output; on the other hand, dual hybrid motor operates with the reverse phase control method and was able to gain higher speed. Such phenomenon brings attractive characteristics of generating either higher speed or larger torque while operating with different control methods. As discussed above, the proposed dual hybrid motor was easily formed by connecting two single hybrid motors, and was able to increase different output performance for certain applications while running the same phase or reverse control method.

\section{CONCLUSION}

Multiple solutions can be easily completed only by adjusting the dimension of elastic blocks degeneracy process. Note that, although the $1^{\text {st }}$ mode for both the longitudinal and torsional vibrators was applied to the case studies in this article, the $2^{\text {nd }}$ modes or even the $3^{\text {rd }}$ mode with higher resonance frequency is likely to be chosen for use. Using a template employed in between the longitudinal and torsional vibrators enabled the reduction of the mutual vibration effect so that the sought resonance frequencies measured by the Impedance Analyzer and calculated in ANSYS are both in good agreement. And the actual driving frequency was approximate to the sought resonance frequency, which was instrumental in design. Because of the structure design of the stator, it was very easy to directly butt each stator of the two single hybrid motors to form a dual hybrid motor. Prototypes of the single and dual hybrid motor with appropriate length of elastic block were developed and driven with a suitable resonance frequency chosen in the searched range of degeneracy respectively. Besides an attempt to increase the output by means of the butted dual hybrid motors, different outcomes of torque and speed are obtained by driving with different control commands. As expected, greater torque and speed was gained in comparison with the single hybrid motor. The maximum torque or speed was improved to nearly two times of the single hybrid motor while using the same phase control or the reverse phase control methods. In particular, the dual hybrid motor operates with reverse phase control command and can reach nearly twice the speed, which was not feasible to obtain due to no summation of speed result while concurrently running two individual single hybrid motors. Further study of the dimension and material of the template as well as verification of its influence will be continued. For example, by connecting the rotor with the gear and belt, it benefits both single and dual hybrid motors. Nonetheless, there needs to be a comprehensive mechanism design of the dual hybrid motor in the case of linking it with another shaft directly, which will be a study that will be tackled in the near future.

\section{ACKNOWLEDGEMENT}

This research was supported by the project of the National Science Council under the grant NSC94-2212-E-033-008 and NSC100-2622-E-033-007-CC2, and sponsored by Soundwide Technology Corp.

\section{REFERENCES}

1 Kurosawa, M. and Ueha, S. Hybrid Transducer Type Ultrasonic Motor, IEEE Transactions on Ultrasonics, Fer- roelectrics and Frequency Control, 38(2), 89-92, (1991). https://dx.doi.org/10.1109/58.68464

2 Nakamura K., Kurosawa, M. and Ueha, S. Characteristics of a Hybrid Transducer-Type Ultrasonic Motor, IEEE Transactions on Ultrasonics, Ferroelectrics and Frequency Control, 38(3), 188-193, (1991). https://dx.doi.org/10.1109/58.79602

3 Nakamura K., Kurosawa, M. and Ueha, S. Design of a Hybrid Transducer Type Ultrasonic Motor, IEEE Transactions on Ultrasonics, Ferroelectrics and Frequency Control, 40(4), 395-401, (1993). https://dx.doi.org/10.1109/58.251288

4 Lin S. Sandwiched Piezoelectric Ultrasonic Transducers of Longitudinal-Torsional Compound Vibrational Modes, IEEE Transactions on Ultrasonics, Ferroelectrics and Frequency Control, 44(6), 1189-1197, (1997). https://dx.doi.org/10.1109/58.656619

5 Tomikawa Y., Adachi K., Aoyagi M., Sagae T. and Takano T. Some Constructions and Characteristics of Rod-Type Piezoelectric Ultrasonic Motors Using Longitudinal and Torsional Vibrations, IEEE Transactions on Ultrasonics, Ferroelectrics and Frequency Control, 39(5), 600-608, (1992). https://dx.doi.org/10.1109/58.156178

6 Wang J. and Guo J. Development of a Radial-Torsional Vibration Hybrid Type Ultrasonic Motor with Hollow and Short Cylindrical Structure, IEEE Transactions on Ultrasonics, Ferroelectrics and Frequency Control, 56(5), 10541058, (2009). https://dx.doi.org/10.1109/tuffc.2009.-1138

7 Yi Y., Seeman W., Gausmann R. and Zhong J. Development and Analysis of a Longitudinal and Torsional Type Ultrasonic Motor with Two Stators, Ultrasonics, 43(8), 629-634, (2009). https://dx.doi.org/10.1016/j.ultras.2005.03.007

8 Sanatobu J., Lee D., Nakamura K. and Ueha S. Improvement of the Longitudinal Vibration System for the Hybrid Transducer Ultrasonic Motor, IEEE Transactions on Ultrasonics, Ferroelectrics and Frequency Control, 47(1), 216221, (2000). https://dx.doi.org/10.1109/58.818764

9 Ishii T., Yamawaki H. and Nakamura K. An Ultrasonic Motor Using Thrust Bearing for Friction Drive with Lubricant, 2013 Joint IEEE UFFC, EFTF and PFM Symposium, 2125, (2013). https://dx.doi.org/10.11-09/ultsym.2013.0051

10 Qiu W., Mizuno Y., Koyama D. and Nakamura K. Efficiency Improvement of Hybrid Transducer-Type Ultrasonic Motor Using Lubricant, IEEE Transactions on Ultrasonics, Ferroelectrics and Frequency Control, 60(4), 786-794, (2013). https://dx.doi.org/10.1109/tuffc.2013.2627

11 Zippo A., Barbieri M., Iarriccio G. and Pellicano F. Nonlinear vibrations of circular cylindrical shells with thermal effects: an experimental study, Nonlinear Dynamics, 99, 373-391, (2019). https://dx.doi.org/10.1007/s11071018-04753-1

12 Zippo A., Barbieri M. and Pellicano F. Temperature gradient effect on dynamic properties of a polymeric circular cylindrical shell, Composite Structures, 216, 301-314, (2019). https://dx.doi.org/10.1007/s11071-018-04753-1 